\title{
A Model for Flow-Induced Acoustics in Corrugated Pipes
}

\author{
Mihaela Popescu ${ }^{1}$ Stein Tore Johansen ${ }^{2}$ \\ Department of Process Technology, Flow Technology, \\ SINTEF Materials and Chemistry, Trondheim, Norway, 7046 \\ Wei Shyy ${ }^{3}$ \\ Department of Aerospace Engineering \\ University of Michigan \\ Ann Arbor, MI, USA
}

\begin{abstract}
Waves and vortex shedding in corrugated pipes during gas injection can produce distinct tonal noise and structural vibration. In this study, we propose a model which couples an acoustic pipe and self-excited oscillation with vortex shedding over the cavity. In the model, the acoustic response of the corrugated pipe is simulated by connecting the lossless medium moving with a constant velocity with a source based on discrete distribution of van der Pol oscillators arranged along the pipe. Our time accurate solutions exhibit dynamic behaviour consistent with that experimentally observed, including the lock-in frequency of vortex shedding and pipe oscillation, and the onset fluid velocity capable of generating the lock-in.
\end{abstract}

\section{Nomenclature}

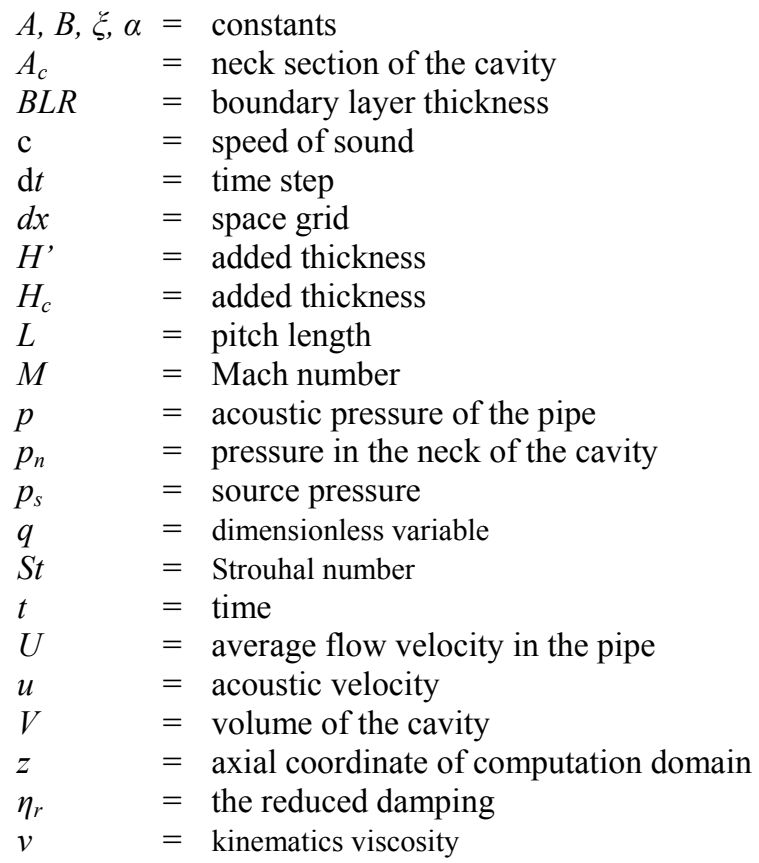

\footnotetext{
${ }^{1}$ Research Scientist, Department of Process Technology, Flow Technology, Sem Sælands vei 2 a, Trondheim, AIAA Member..

${ }^{2}$ Principal Scientist, Department of Process Technology, Flow Technology, Sem Sælands vei 2 a, Trondheim.

${ }^{3}$ Clarence L. "Kelly” Johnson Collegiate Professor and Chair, Fellow AIAA, weishyy@umich.edu

American Institute of Aeronautics and Astronautics 092407
} 
$\begin{array}{ll}\rho_{0} & =\text { reference density } \\ \omega & =\text { frequency } \\ \omega_{r} & =\text { angular frequency of the resonator }\end{array}$

\section{Introduction}

$\mathrm{R}^{\mathrm{i}}$ isers are pipes that facilitate fluid flow between sea installations and surface facilities located on drill platforms. The riser can be either free standing or structurally flexible, or top tensioned and rigid. The most popular riser used in deepwater is flexible. One of the main characteristics of a flexible pipe is its low relative bending to axial stiffness, achieved through use of a number of layers of different materials in the pipe wall fabrication. These layers are able to slip past each other when under the influence of external and internal loads. The structural and geometrical complexity of riser is exemplified in Figure 1. The carcass is the innermost layer, which is the essential layer because it takes the loads induced by internal pressure and the longitudinal force. It is commonly made of stainless steel flap strips that are formed into interlocking profile. The cavity appears between two consecutive interlocking strips.(see Figure 2).

The flexible risers often experience the phenomenon of "singing": large pressure fluctuations within the riser that can be heard as acoustic tones. The problem can be attributed to flow induced pulsations that are generated on the inner corrugated layer of the flexible riser. When the vortex shedding frequency excites acoustic natural frequency of the pipeline, resonance between structural vibration and vortex shedding appears and this is known as lock-in. The minimum fluid velocity for which lock-in frequency appear is referred to as the onset velocity.

This study was initiated to help shed light on the singing phenomena with the goal of developing a capability to simulate the vortex shedding and associated waves in corrugated pipes during gas injection. . The shedding frequency of vortices can be characterized by the Strouhal number. Ziada et $\mathrm{al}^{2}$ established that the vibrations occur over a certain range of Strouhal numbers. Nakamura and Fukamachi ${ }^{14}$ showed that the frequency of the loudest sound from corrugated pipe is proportional to the flow velocity. In other word the Strouhal number can be approximated like constant.

$$
S t=\omega \cdot L / U
$$

Where $\omega$ is the frequency that characterizes the flow, $L$ is the pitch length and $U$ is the average flow velocity. Weaver and Ainsworth ${ }^{3}$ showed that the Strouhal number is typically larger than 0.45 for the maximum vibration amplitude. This value is in agreement with the results of Gerlach ${ }^{5}$, Bass and Holster ${ }^{6}$ and Klaeui ${ }^{7}$

Flow-excited and self-sustained resonance is observed in a large number of engineering applications; a substantial literature exists in this topical area. To our knowledge, the first work on sound propagation in corrugated pipes was done by Burstyn ${ }^{8}$ in 1922 . He suggested that the corrugations act as "numerous lips". Independently, Cermak $^{9}$ experimentally investigated the phenomena based on a pipe of $10 \mathrm{~mm}$ outer diameter and $0.5 \mathrm{~m}$. He noticed that it is difficult to excite the fundamental tone. Silverman ${ }^{10}$, Crawford $^{11}$ and Cadwell ${ }^{12}$ made connection between frequency of the sound and flow velocity. Popescu and Johansen ${ }^{13}$, Nakamura and al ${ }^{14}$, and Kristiansen ${ }^{15}$ reported the connection between sound emitted in a tube and shear layer instability resulting from the flow over the corrugation. They suggested that the interaction between the fluid flow with the cavities is responsible for the resonance and noise. In a more generic context, How ${ }^{16}$ demonstrated theoretically that shear layer/cavities interaction results in two types of resonance sources: monopole and dipole. Hémon et $\mathrm{al}^{17}$ present an experimental and theoretical study of the pressure oscillations generated by the flow over deep cavity. A review of recent advances in understanding, modelling and controlling oscillations of flow past a cavity has been given by Rowley and Williams ${ }^{18}$.

Rockwell and Schachenmann ${ }^{19}$ Error! Reference source not found. provided the first measurements of the physical behaviour of an unsteady shear layer along the mouth of a circular cavity at the end of a long pipe, including both locked-in and non-locked-in state. They showed that during lock-in, the magnitude of the fluctuating velocity due to acoustic resonance is of the same order as that associated with the hydrodynamic fluctuations.

There is also work done specifically in modelling of the fluid flow over cavities. Debut ${ }^{20}$ et al. presented a phenomenological model of the flow around corrugation. They proposed a way to describe feedback mechanism of the acoustics-cavity interactions. Tam and Block ${ }^{21}$ derived a mathematical model of acoustic cavity to couple the 
cavity tones, shear layer instability, and acoustic feedback to help understand the tone generation mechanism for flow over the cavities. This model includes the effect of the shear layer thickness, which is important.

The purpose of this work is to develop a computational model, in a simplified one-dimensional framework, to capture the main dynamic characteristics described above. In particular, we address the coupling mechanism between acoustic oscillations and the fluid flow over a series of cavities. In the following, we first present the mathematical model of a coupled mechanism between singing in the corrugated pipe and flow-induced oscillations such as vortex shedding. The model is based on a wave equation representing the acoustic field, coupled with selfexcited equations of the Van der Pol type accounting for fluid-induced oscillations around cavities. The aeroacoustic coupling is expressed in terms of the gradient pressure source. The frequency that characterizes the vortex shedding can be determined by computing the flow over a single cavity ${ }^{13}$ in the form of the Strouhal number. We assess the outcome of the model via direct experimental comparison, in particular, lock-in frequency and onset fluid velocity.

\section{Computational Model}

The acoustic field and the aerodynamic flow field strongly interact in resonance conditions. The acoustic flow velocity influences the roll-up of the vortices when they are shed to downstream. At the same time the aerodynamic flow field also influences the acoustic flow field: energy is transferred from the former to the latter flow field under specific physical conditions: see Figure 3. Identification of these conditions and mathematical simulation of this coupled process is a main objective for the present paper.

The one dimensional flow-acoustic model consists of two key components: (i) the acoustic pipe; (ii) self-excited oscillators representing the vortex excitation caused by the flow over corrugation.

\section{A. Model for pressure in a corrugated pipe}

A simple and reasonable model which utilizing the concept of mechanical oscillator to represent shear layer instability is described by the following equation ${ }^{17}$ :

$$
\ddot{p}_{s}+2 \eta_{r} \omega_{r} \dot{p}_{s}+\omega_{r}^{2} p_{s}=\xi \omega_{r}^{2} p
$$

Where

- $p_{s}$ is the acoustic pressure in the cavity,

- $\quad p$ pressure in the neck

- $\omega_{r}$ is angular frequency of the resonator and $\omega_{r}=c \sqrt{\frac{A_{c}}{V\left(H_{c}+2 H^{\prime}\right)}}$, where $A_{c}$ is neck section, $V$ is volume of the cavity, $H_{c}$ thickness of the neck, $H^{\prime}$ added thickness (correction) of the neck

- $\quad \eta_{r}$ is the reduced damping, $\eta_{r}=\omega^{2} \omega_{r} \frac{V}{2 \pi c^{3}}$, where $\omega$ is angular frequency and $\mathrm{V}$ is the volume of the cavity

For the corrugated pipe, the instability of the shear layer is strongly influenced by the acoustic inside the pipe ${ }^{13}$. In this case the driving force in the cavity is natural frequency of the pipe. Consequently, the pressure from the neck is replaced by the derivative of acoustic pressure from pipe because, in accordance with Howe's analogy, the acoustic energy generation can be deduced based on the acoustic velocity in the source region ${ }^{22}$. The section neck is replaced by the section of the corrugation inside the pipe. Because the principal resonator is the pipe we will interchange the position of $\omega$ with $\omega_{r}$.

The equation that describes the shear instability does not present a self-sustained regime. Hence, we need further modification of equation (2). We consider a model that was well studied in the dynamical systems literature ${ }^{5,6,7}$ the van der Pol oscillator, which is a nonlinear oscillator, and, like the cavity in a self-sustained regime, has negative damping at low amplitudes and positive damping at high amplitudes. A way to transform equation (2) into a van der Pol type is to replace the reduced damping as follows:

$$
\eta_{r} \rightarrow A \eta_{r}\left\{\left(\frac{p_{s}}{B \rho_{0} U^{2}}\right)^{2}-1\right\}
$$

As a result, the pressure in the corrugated pipe can be described by the equation: 


$$
\ddot{p}_{s}+2 \eta_{r} A\left\{\left(\frac{p_{s}}{B \rho_{0} U^{2}}\right)^{2}-1\right\} \omega \dot{p}_{s}+\omega^{2} p_{s}=\xi \omega p^{\prime}
$$

where $p^{\prime}$ is the space derivative of the acoustic pressure, and

$$
\eta_{r}=\omega_{r}^{2} \omega \frac{V}{2 \pi c^{3}}
$$

The excitation term from is defined similar to that given by Hémon et al ${ }^{17}$

$$
\varsigma=\alpha \frac{\omega}{\omega_{r}} \frac{|p|}{\left|p+p_{s}\right|}
$$

The source is the vortex excitation caused by the flow over the corrugation. In our feed-back model this source is represented by self-excited oscillators of the Van der Pol type, with saturation expressed in terms of amplitude. As Facchinetti ${ }^{23}$ at al. showed that some three dimensional feature of the vortex shedding in the near wake of stationary slender bluff bodies in stationary flow can be described qualitatively and quantitatively by a low order dynamical model, formed by van der Pol oscillators along the spanwise extent of the structure.

$$
\frac{\partial^{2} q}{\partial t^{2}}+\varepsilon \omega\left(q^{2}-1\right) \frac{\partial q}{\partial t}+\omega^{2} q-v \frac{\partial^{3} q}{\partial t \partial z^{2}}=0
$$

where $\mathrm{z}$ is the direction of the axis of the pipe, $q$ is a dimensionless variable describing the wake flow, $v$ is a diffusion parameter, $\omega$ is the vortex shedding angular frequency and, $\varepsilon$ is a positive parameter of the van der Pol oscillator. The authors demonstrate that the diffusion interaction is able to model vortex shedding in shear flow.

Taking into account equation (4) and (7) we will obtain the final form of the source equation:

$$
\ddot{p}_{s}+2 \eta_{r} A\left\{\left(\frac{p_{s}}{B \rho_{0} U^{2}}\right)^{2}-1\right\} \omega \dot{p}_{s}+\omega^{2} p_{s}-v \frac{\partial^{3} p_{s}}{\partial t \partial z^{2}}=\xi \omega c p^{\prime}
$$

\section{B. Acoustic pressure in the pipe}

In the pipe the acoustic behavior can be described as the lossless medium moving with a constant velocity linear wave equation:

$$
\left\{\begin{array}{l}
\frac{\partial\left(\rho_{0} u\right)}{\partial t}+U \frac{\partial\left(\rho_{0} u\right)}{\partial z}+\frac{\partial p}{\partial z}=F(z, t) \\
\frac{\partial p}{\partial t}+U \frac{\partial p}{\partial z}+\rho_{0} c_{0}^{2} \frac{\partial u}{\partial z}=0
\end{array}\right.
$$

where the source term from previous equation is

$$
F(z, t)=G \frac{\partial p_{s}}{\partial z}
$$

where $p_{s}$ is the pressure variation caused by the source cavities and $G$ is a constant that depend on the opening of the cavity because the feedback mechanism depend on the opening of the cavity: $G$ is the ratio between opening of the cavity and the length of the pitch.

The acoustic formulation represented by Eq.(9) is coupled with $\mathrm{N}$ equations for the source variables formulated such that each source oscillates with characteristic frequency $\omega$. The position and the number of the source is determined by the position and the number of the corrugation in the pipe. 
The characteristics of the cavity, the shape and the volume of the cavity will control mainly the limits of the pressure field. This roll is plaid by the B in the Van der Poll equation. Unfortunately, currently, we do not have a definitive value of parameter B. Therefore, we take the value of B like the ratio between the volumes of the cavity to the volume of the pipe which has the length of the cavity opening.

Equations (2) and (9) are solved using high order schemes: Optimized Prefactored Compact finite volume (OPCfv) scheme $^{30}$ for discretization in space, and Runge-Kutta ${ }^{31}$ for time stepping. These techniques are designed to handle wave propagation with source terms, and are capable of producing solutions of low numerical dispersion and dissipation, as well as satisfactorily honoring the conservation laws. In Eq. (7), two coefficients, A, and B, need to be prescribed. They are discussed in the following.

\section{Coefficient 'A'}

Tam $^{21}$ suggested that the instability of free shear layer has an important contribution to the driving mechanism of cavity oscillation. The unstable behavior of this phenomenon constitutes an essential ingredient in understanding the origin of the pressure oscillations.

Huerre and Monkewitz ${ }^{24}$ demonstrated that the mixing layer with a small velocity ratio was subjected to connectivity stability. Hémon et $\mathrm{al}^{17}$ showed that in case of cavity, the streamwise direction is bounded by the edges, which leads to perturbation generated by the periodic impingent of the vortices. This will determine to generate the self sustained shear layer oscillations, the frequency of which is related to the distance between the upstream and downstream edges ${ }^{13}$. For flow over a cavity, the bottom part of the boundary layer develops into a shear layer and the boundary layer vortices may roll up into discreet vortex ${ }^{13,26}$. The process is similar to a Kelvin-Helmholtz instability ${ }^{27}$.

The dimension of the boundary layer in this case is directly connected to the level of shear layer instability, and accordingly, to the thickness of the shear layer. As we know the shear layer of an oscillating cavity is turbulent. In the instability zone only a part of energy associated to the pressure fluctuation radiated like a sound ${ }^{25}$. In this flow region pressure fluctuations are dominated by the inertial effect rather than the compressibility. Hence by increasing boundary layer will tend to prevent the occurrence of lock-in regimes. We can conclude that to be able to have a stronger signal from the source we need that boundary layer to be thinner. Finally we can notice that boundary layer control the way in which the sound pressure flow through system, which is the roll of coefficient A in the Van der Poll Eq. (8)

So we notice in Figure 5 the parameter A from the equation that describe the source sound have the same behavior like the thickness of boundary layer. An approximation of the A parameter should be connected to the thickness of boundary layer. In this work we propose

$$
\mathrm{A}=0.5 * \mathrm{BLR}
$$

where BLR is the ratio between the boundary layer thickness and the radius of the pipe. If BLR is close to one, we deal with only turbulent flow. In this case the feedback mechanism doesn't exist, and the singing phenomenon doesn't appear.

\section{Coefficient 'B'}

Krishnamurty ${ }^{28}$ and Rossiter $^{1}$ recognized that the interaction between the oscillating shear layer and the trailing edge of the cavity produced intense acoustic disturbance. Experimental evidence leaves no room for doubt concerning the existence of this acoustic source. Heller and Bliss ${ }^{29}$ used the water table visualization to observe the sequence events which took place during a typical oscillation cycle. They found that the compression wave (shock wave) produced at the trailing edge of the cavity extended from inside the cavity all the way to the supersonic outside flow. Before they are modified by the outside mean flow, observations clear indicate that the pressure disturbances inside and outside the cavity are in phase. In consequence one of main roll in the level of pressure level sound is determined by the interaction between shear layer and trailing edge and the capacity of resonance

\section{Results and Discussion}

A main interest of the acoustic simulation of the corrugated pipes is to predict the lock-in behavior and the onset flow rate. We assess the computational outcome by utilizing available field measurements from gas transport systems. 
To obtain the value of the frequency that is characteristic for a certain cavity and for a certain velocity, we compute Strouhal number using computation fluid dynamics around a single cavity, using the periodic boundary condition.

Experimental measurements for two different pipe and flow configurations are available ${ }^{15,34}$ and will be used to evaluate the present computational model. Table 1 summarizes the geometric and flow parameters and Figure $\mathbf{4}$ and Figure 10 show the schematic configurations of the two cases. In all cases the Mach number is less than one.

In Case I, as established by Popescu et $\mathrm{al}^{13}$ based on the computation of the flow around a single cavity with periodic boundary condition and confirmed by the experimental data ${ }^{15}$, the Strouhal number (Eq. (1)) is 0.61 . In Figure 6 a limit cycle of the source is presented for the fluid velocity of $18 \mathrm{~m} / \mathrm{s}$. As expected, the limit circle forms after a while when the coupling between acoustics and fluid flow becomes balanced. Figure 7 presents the prediction of the resulting acoustic wave in the pipe for the case of $18 \mathrm{~m} / \mathrm{s}$ average fluid velocity, caused by the vortex excitation around the cavity. In this case we deal with self sustained regime, namely, a singing pipe. This figure shows that the amplitude of the acoustic pressure remains constant, which indicate stability of the acoustic system (singing).

The simulation was done for flow between 5.5 and $20 \mathrm{~m} / \mathrm{s}$, but the singing was observed only for velocity higher than $6 \mathrm{~m} / \mathrm{s}$. The experimental results recorded indicate that singing starts at a fluid speed of $3.8 \mathrm{~m} / \mathrm{s}$, which correspond to the one wave length. In other words, the model doesn't capture the capacity of singing if the length of the pipe is not at least equal to one and a half wave length.

Figure 8 a shows the overall trend of the oscillating frequency of the system as the velocity is increased. We notice different stages corresponding to the lock-in phenomenon. The various stages are separated by jumps in frequency. The prediction agrees well with the experimental data in all cases, with the maximum error of $1.25 \%$.

Studying the evolution of pressure amplitude in Figure 8b, we first notice that amplitude of the pressure wave, which characterizes the flow, are high, and that overall, the higher the resonance frequency, the higher the fluid pressure. It is also noticed that the pressure first increases, then passes through a maximum, and finally drops suddenly as the flow velocity increases. Similar phenomena were also reported by Debut et al ${ }^{33}$, as shown in Figure 9.

The pressure oscillation in the pipe is driven by vortex shedding from the cavity and the shear layer instability. When the shear-layer frequency coincides with a natural frequency of the pipe, the acoustic oscillation of the tube is resonantly excited. The observed frequency lock-in indicates that the shear-layer frequency is influenced by the acoustic oscillation: the two mechanisms strongly influence each other. Thus, the oscillations under discussion are a consequence of a coupled fluid-acoustic system. The acoustic field responds to the shear layer instability (vortex shedding frequency) and the geometry of the tube (resonance). The fluid flow is influenced by the acoustic field; the shear layer frequency is influenced by the acoustic frequency, and adapts to it. Consequently, both acoustic and shear layer frequencies tend to match each other. The acoustic-fluid system is characterized by one dominant frequency over a range of the fluid speed. The frequency jump can be interpreted as the system tending a minimum in the overall energy state.

In Case II, the simulation was done for the fluid velocity from $3 \mathrm{~m} / \mathrm{s}$ to $5 \mathrm{~m} / \mathrm{s}$, in a pipe of $25 \mathrm{~m}$ long. The Strouhal number, according to Dhainaut work ${ }^{34}$, is equal to one. As shown in Figure 11, for a fluid velocity of $3 \mathrm{~m} / \mathrm{s}$, the acoustic wave decays in time, indicating that singing cannot be sustained at this velocity. In fact, singing is not attained computationally for fluid velocity lower than $3.5 \mathrm{~m} / \mathrm{s}$. In Figure 12 shows the simulation for a fluid velocity equal to $3.5 \mathrm{~m} / \mathrm{s}$, we see that acoustic wave has stable amplitude, which is the predicted on-set velocity. This predicted value is the same as that reported in the experimental data ${ }^{34}$. Figure 13 shows the general trends of the oscillating frequency of the system versus the fluid velocity. The numerical simulation and the experimental data again agree with each other favourably.

\section{Summary and Conclusion}

In this work we have developed a one dimensional model, which couples the vortex shedding mechanism and the acoustic oscillation in a corrugated pipe. The model consists of two key components:

(i) an acoustic treatment modeled as a lossless medium moving with a constant velocity, in the form of a linear wave equation;

(ii) an oscillator model, based on the Van der Pol type equation, which takes into account the vortex shedding in shear flow and excitation and damping according to the shear layer instability theory

The model was validated against two experimental cases. The solution demonstrates that can capture the capacity of singing only for pipes longer than 1.5 wave length of the acoustic wave. Furthermore, the model is capable of predicting the lock-in frequency as well as the onset fluid velocity. 


\section{Acknowledgments}

The work was funded by the Norwegian Research Council, together with the industrial partners Wellstream, Technip and StatoilHydro. Special thanks go to project manager Øyvind Hellan for the support given during this work.

\section{References}

${ }^{1}$ Rossiter, J.E., "Wind-tunnel experiments on the flow over rectangular cavities at subsonic and transonic speed," Technical Report 3438, Aeronautical Research Council Reports and Memoranda, 1964

${ }^{2}$ Ziada, S., M.Eng, Asme, M., and Buhlmann, E.T., "Flow induced vibration in long corrugated pipes," C416/010@IMechE, 1991, pp. $417-426$

${ }^{3}$ Weave, D.S., and Ainsworth, P., "Flow induced vibration in bellows", International Symposium on Flow-Induced Vibration and Noise, Chicago, 1988, pp.205-214

${ }^{4}$ Gerlach, C.R., "Flow-induced vibration of metal bellows," J. Engineering for industry; 91(4): 1969, pp.1196-1202

${ }^{5}$ Gerlach, C.R., "Vortex excitation of metal bellows," J. Engineering for industry;94(1): 1972, pp. 87-94

${ }^{6}$ Bass, R.L. and Holster, J.L., "Bellows vibration with internal cryogenic flows," ASME J. Engineering for industry; 91(1): 1972, pp.70-75 1987

Klaeui, E., “Jet: vibration tests on calorimeter bellows.” Report No.3554/1512, Sulzer Bros. Ltd., Winterthur, Switzerland,

${ }^{8}$ Burstyn, W., "Eine neue pfeife", Zeitrich für Technique Physics; 3: 1922, pp.179-180

${ }^{9}$ Cermak, P., "Über die tonbildung bei metallschläuchen mit eingedrücktem spiralgang”, Physicalische Zeitschrift; 23, 1922, pp.394-397

${ }^{10}$ Silverman, M.P., Cushman, G.M., "Voive of the dragon: the rotating corrugated resonator", European Journal of physics; 10: 1989, pp.298-304

${ }^{11}$ Crawford, F.S., "Singing corrugated pipes", American Journal of Physics; 42: 1974, pp.278-288

${ }^{12}$ Cadwell, L.H., "Singing corrugated pipes", American Journal of Physics; Vol.62(3), 1994, pp.224-227

${ }^{13}$ Popescu, M. and Johansen, S.T. "Acoustic Wave Propagation in Low Mach Flow Pipe", AIAA paper,08- 95691, 46th AIAA Aerospace Sciences Meeting and Exhibit, 2008

${ }^{14}$ Nakamura, Y. and Fukamachi, N., "Sound generation in corrugated tubes," Fluid Dynamics Research, North Holland; Vol.7, 1991, pp.255-261

${ }^{15}$ Kristiansen, U.R. and Wiik, G.A., "Experiments on sound generation in corrugated pipe with flow", J.Acoust. Soc. Am; Vol.121(3), 2007, pp.1337-1344

${ }^{16}$ Howe, M.S., "Mechanism of sound generation by low Mach number flow over a wall cavity", J. Sound and Vibration; Vol.73, 2004, pp.103-123

${ }^{17}$ Hémon, P, Santi, F., Amandolèse, X., "On the pressure oscillation inside a deep cavity excited by a grazing airflow", European j. Mech B/fluids, Vol.23, 2004, pp.617-632

${ }^{18}$ Rowley, C.W., Williams, D.R., "Dynamic and control of high Reynolds number flow over open cavities", Annu. Rev. Fluid Mech., Vol.38, 2006, pp.251-276

${ }^{19}$ Rockwell, D., Schachenmann, A., "The organized shear layer due to oscillations of a turbulent jet through an axisymmetric cavity," J.Sound and Vibration, Vol.87, 1983, pp. 371-382

${ }^{20}$ Debut, V., Antunes, J., Moreira, M., "A phenomenological model for sound generation in corrugated pipes", ISMA, 2007

${ }^{21}$ Tam, C.K.W., and Block, P.J.W. , "On the tones and pressure oscillations induced by the flow over rectangular cavities," $J$. Fluid Mechanics; 89: pp.373-399, 1978

${ }^{22}$ Hirschberg, A., (1995) “Aeroacoustics of wind instruments. In Mechanics of musical instruments", CISM courses and Lectures. Springer-Verlag

${ }^{23}$ Facchinetti, M.I., Langre, de E., Biolley, 2002, "Vortex shedding modeling using diffusive van der Pol oscillators, Mécanique des Fluides, Série II b, 2002, pp.1-6

${ }^{24}$ Huerre, P. and Monkewitz, P.A., "Absolute and convective instability of the hyperbolic-tangent velocity profile, ”. J.Fluid Mech, Vol.159, 1985, pp.151-168

${ }^{25}$ Roger, M., Charbonnier, J.M., “Applied aero-acoustics: prediction methods", von Karman Institute for fluid dynamics, lecture Series 1996-04, 1996

${ }^{26}$ Tietjens, O., Strömungslehre, 1st Ed., Vol. 2, Springer-Verlag, Berlin, pp. 1970, 105-109

${ }^{27}$ van Dommelen, L.L., Unsteady boundary layer separation, Ph.D. thesis, Cornell University, 1981 (unpublished).

${ }^{28}$ Krishnamurty, K, "Acoustic radiation from two-dimensional rectangular cutouts in aerodynamic surface," N.A.C.A. Tech. Note, no.3487, 1955

${ }^{29}$ Heller, H.H. and Bliss, D.B., "The physical mechanism of flow induced pressure fluctuations in cavities and concepts for their suppression," A.I.A.A. Paper, no.75-491, 1975

${ }^{30}$ Popescu, M., Shyy, W, Garbey, M., "A Study of Dispersion-Relation-Preserving and Optimized Prefactored Compact Schemes for Wave Equation”. J. Comput. Phys.; 210 (5): 2005, pp.705-729. 
${ }^{31}$ Hu, F.Q., Hussaini M.Y, and Manthey J., "Low Dissipation and Dispersion Runge-Kutta for Computational Acoustics”. $J$. Comput. Phys.; 124: 1996, pp.177-191.

${ }^{32}$ Reinen, T.A., "Singing riser: overwie," SINTEF, Singing riser 805016, Trondheim, 2007

${ }^{33}$ Debut, V., Antunes, J., Moreira, M., "Experimental Study of the flow-excited acoustical lock-in in a corrugated pipe", ICSV14, Cairns, Australia, 2007

${ }^{34}$ Dhainaut, M, "CFD modelling activity for the Singing riser project; Part II: real case simulation", SINTEF, Singing riser 805016, Trondheim, 2005

\begin{tabular}{|l|l|l|}
\hline Characteristics & Case I & Case II \\
\hline Pipe internal diameter & $2.54 \times 10^{-2} \mathrm{~m}$ & $3.91 \times 10^{-1} \mathrm{~m}$ \\
\hline Corrugation pitch & $5.3 \times 10^{-3} \mathrm{~m}$ & $2.366 \times 10^{-2} \mathrm{~m}$ \\
\hline Cavity depth & $3.11 \times 10^{-3} \mathrm{~m}$ & $7.66 \times 10^{-3} \mathrm{~m}$ \\
\hline Cavity length & $3.12 \times 10^{-3} \mathrm{~m}$ & $7.76 \times 10^{-3} \mathrm{~m}$ \\
\hline Pipe length & $6.15 \times 10^{-1} \mathrm{~m}$ & $25.0 \mathrm{~m}$ \\
\hline Reference density & $1.225 \mathrm{~kg} / \mathrm{m}^{3}$ & $100 \mathrm{~kg} / \mathrm{m}^{3}$ \\
\hline
\end{tabular}

Table 1. Pipe and flow configurations considered

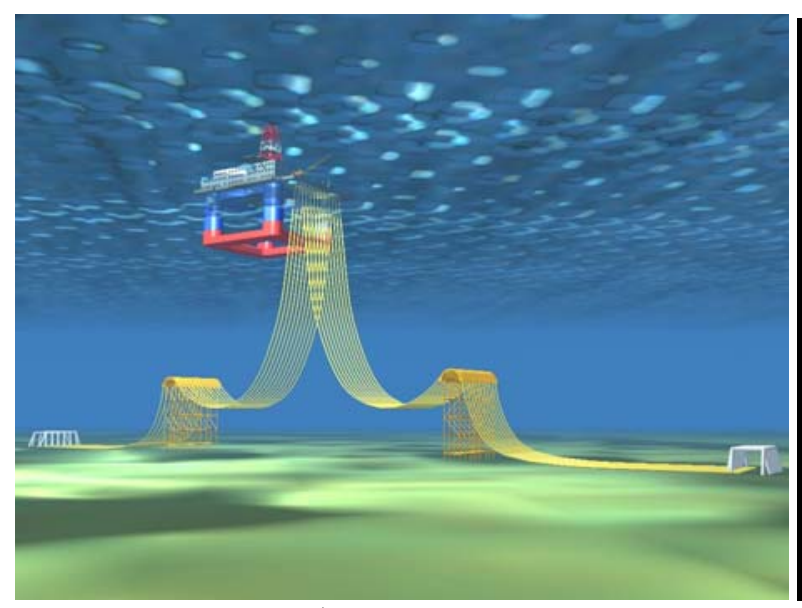

a)

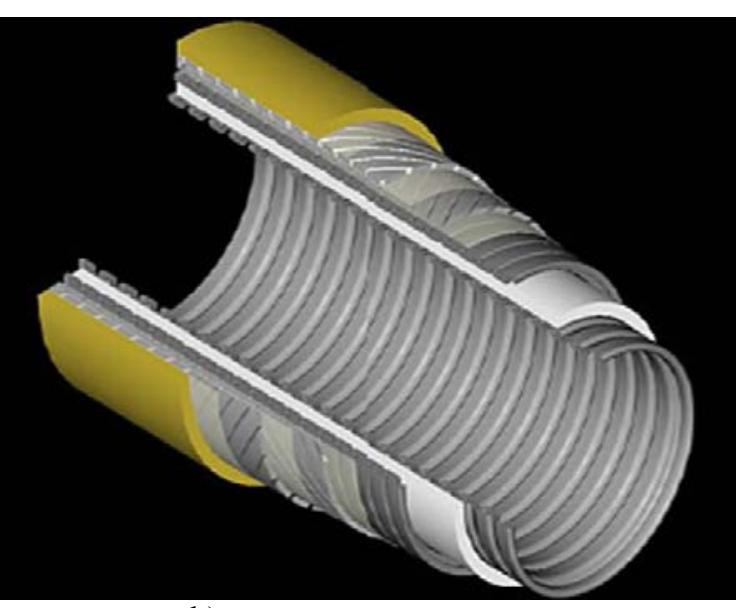

b)

Figure 1. Flexible riser: a) General view of offshore flexible riser system; b) The structure of flexible riser 

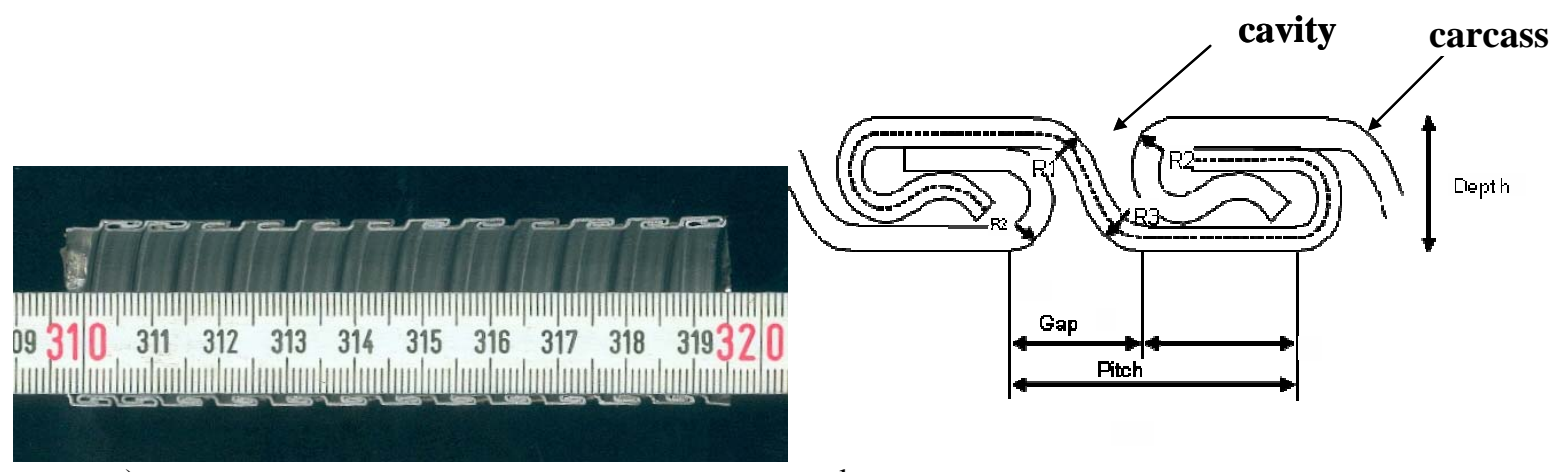

a)

b

Figure 2. Carcass: example of real carcass; b) the scheme of the carcass

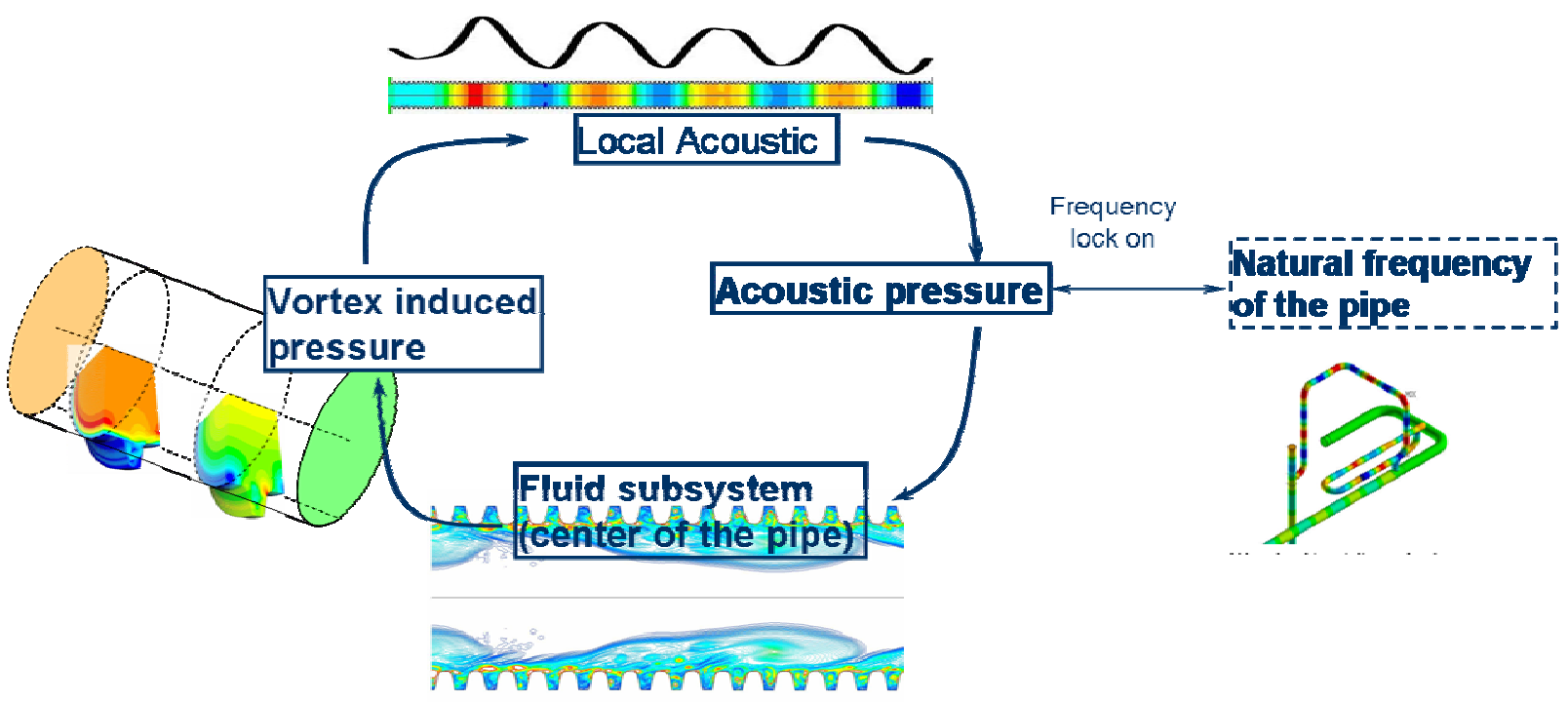

Figure 3. Feedback mechanism between flow and the acoustic field 


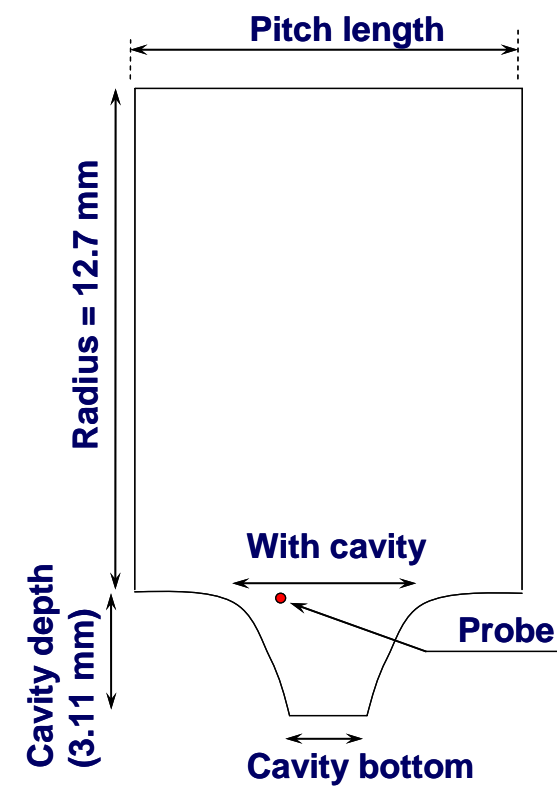

a)

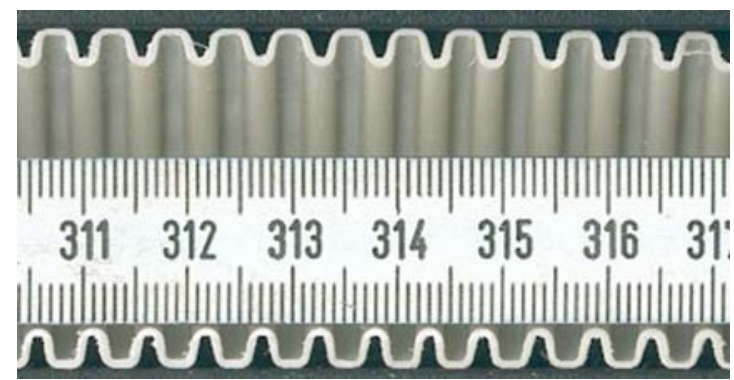

b)

Figure 4. General description of the corrugation for the pipe in Case I: a) geometrical details of the corrugation; b) carcass.

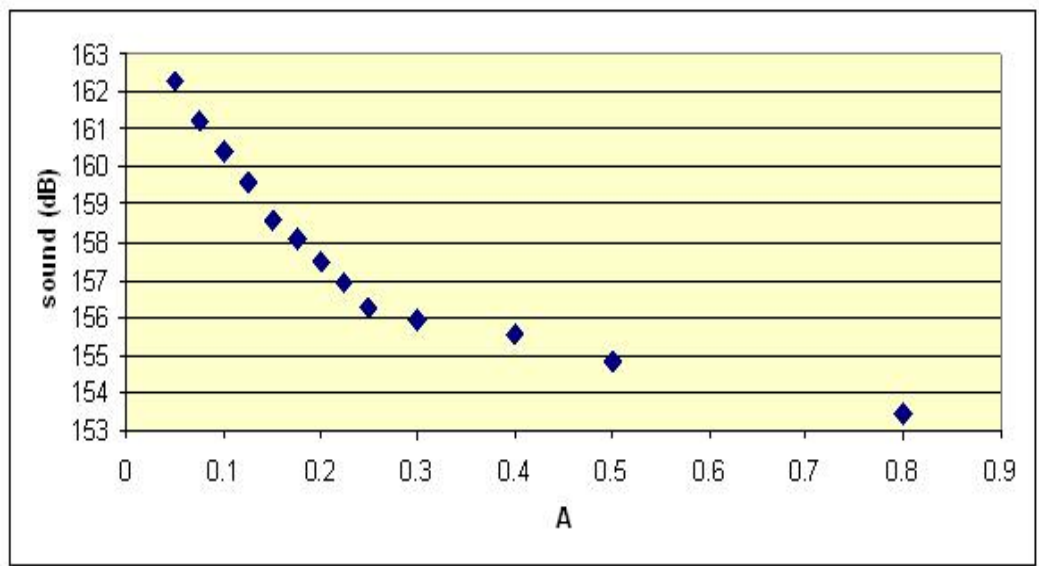

Figure 5. Evolution of the pressure versus to the parameter $\mathrm{A}$ 


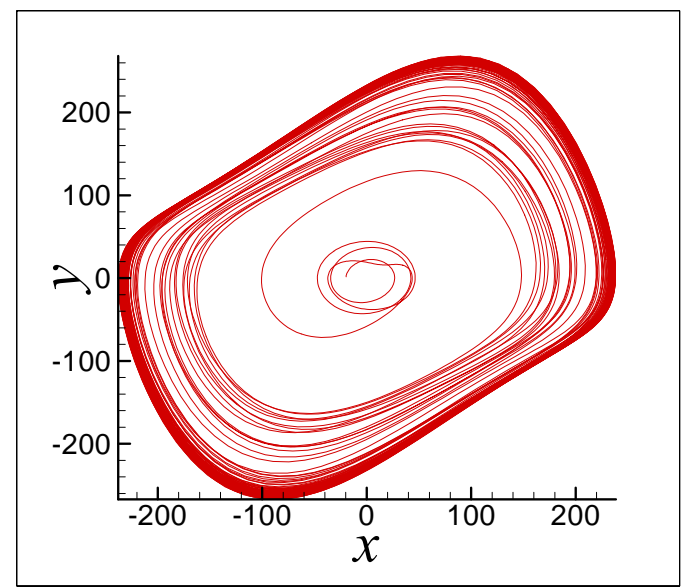

Figure 6. Stable limite cycle of Case I $: \mathrm{x}=$ source pressure, $\mathrm{y}=$ derivative of source pressure
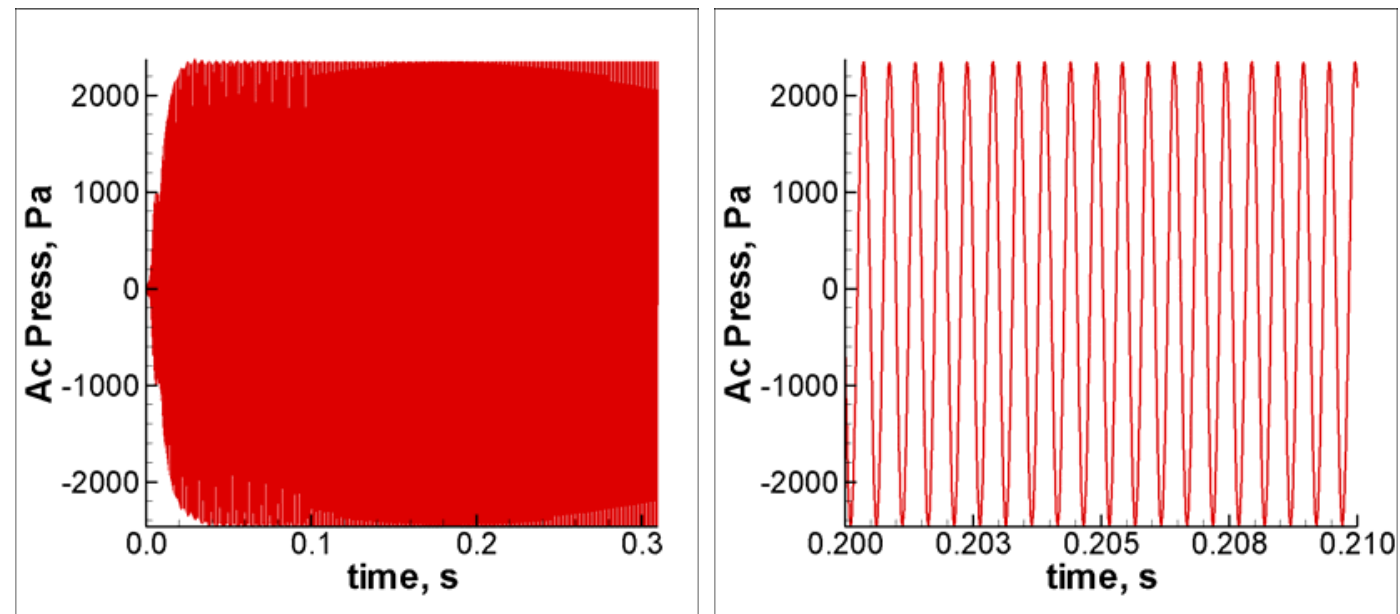

a)

b)

Figure 7. Acoustic pressure of the pipe in the point $x=0.203 \mathrm{~m} ; \mathrm{u}=18 \mathrm{~m} / \mathrm{s}$ for Case $\mathrm{I}$ : a) $\mathrm{t} \in(0,0.31)$; b) Detailed cyclic pattern: $\mathbf{t} \in(\mathbf{0 . 2}, \mathbf{0 . 2 1})$;
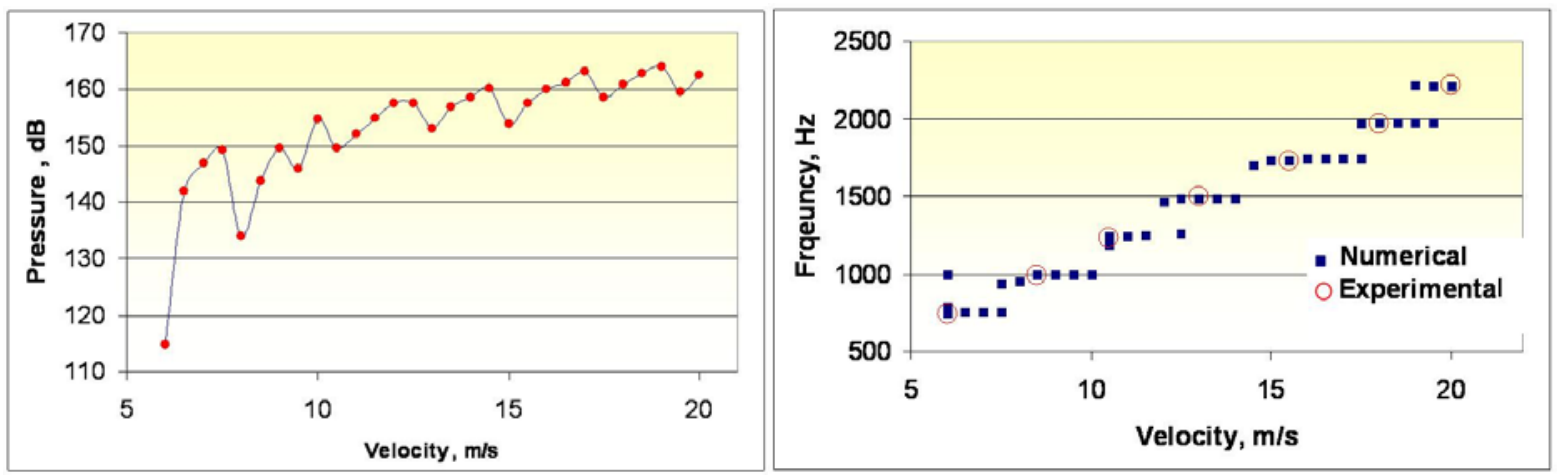

Figure 8. Evolution of frequency (left) and amplitude (right) versus mean flow; Case I 


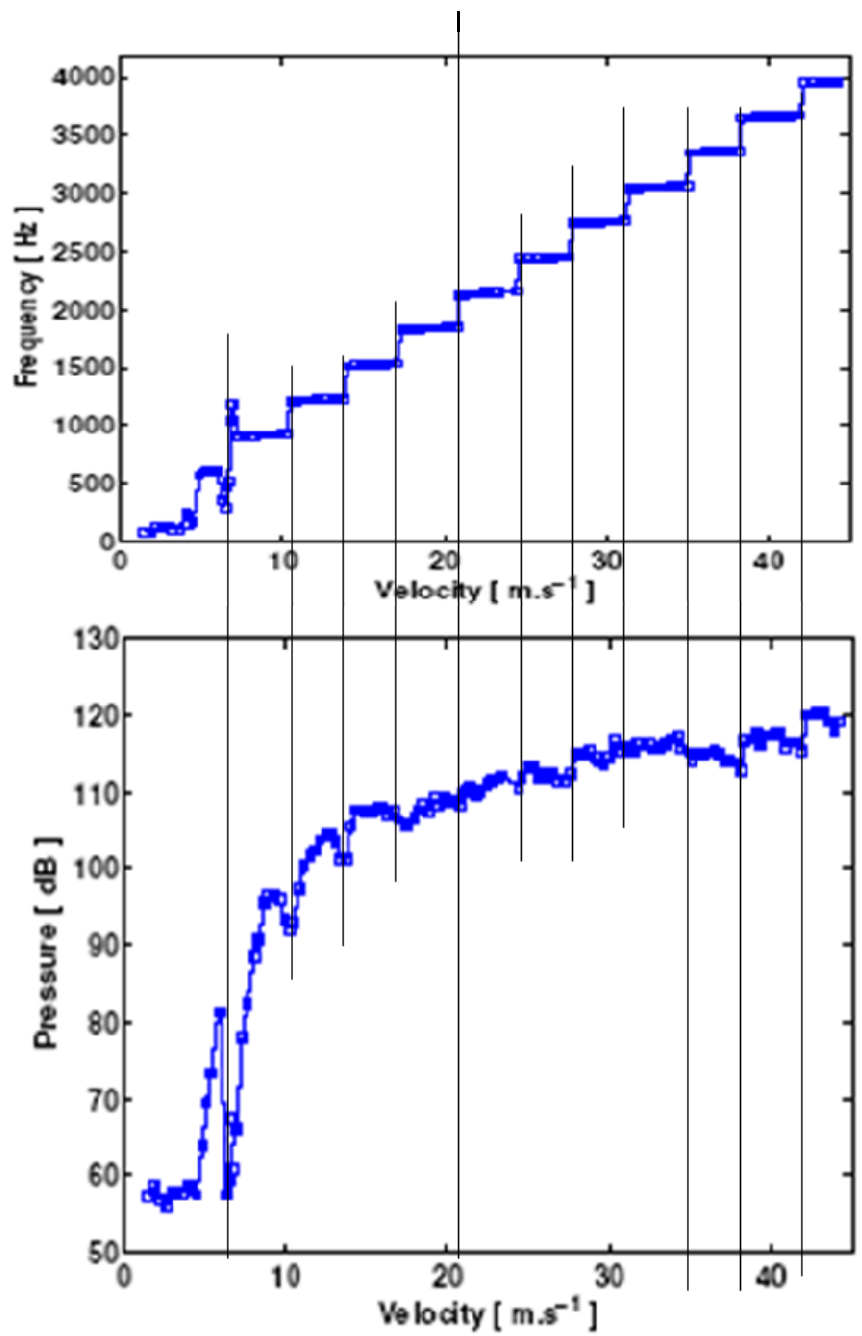

Figure 9. Experimental results in a corrugated pipe done by Debut et al. ${ }^{33}$ : Evolution of dominant frequency and pressure versus velocity.

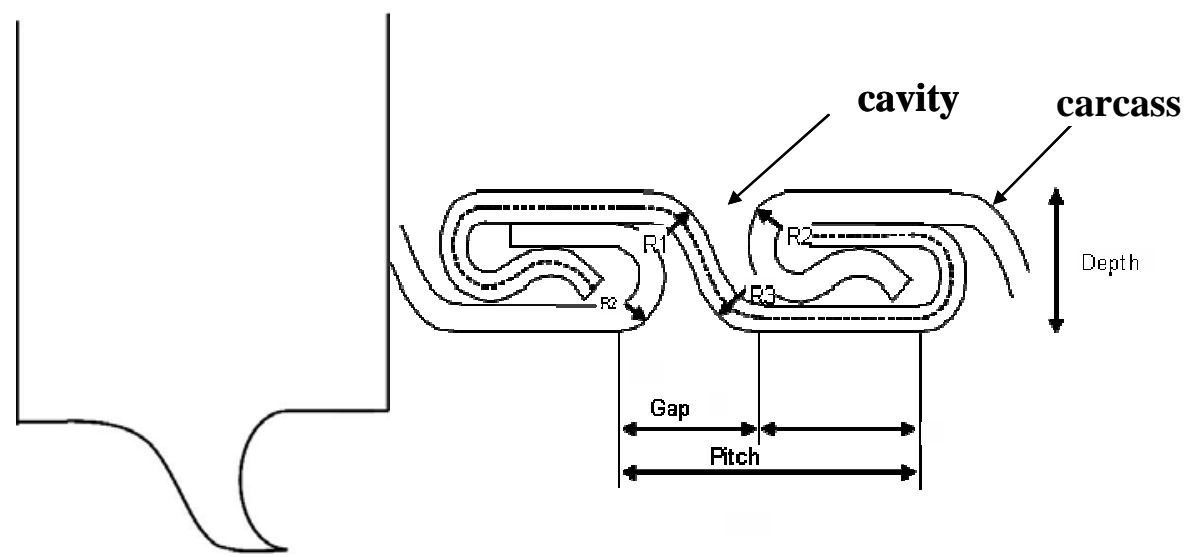

Figure 10. General description of the corrugation for the second pipe 

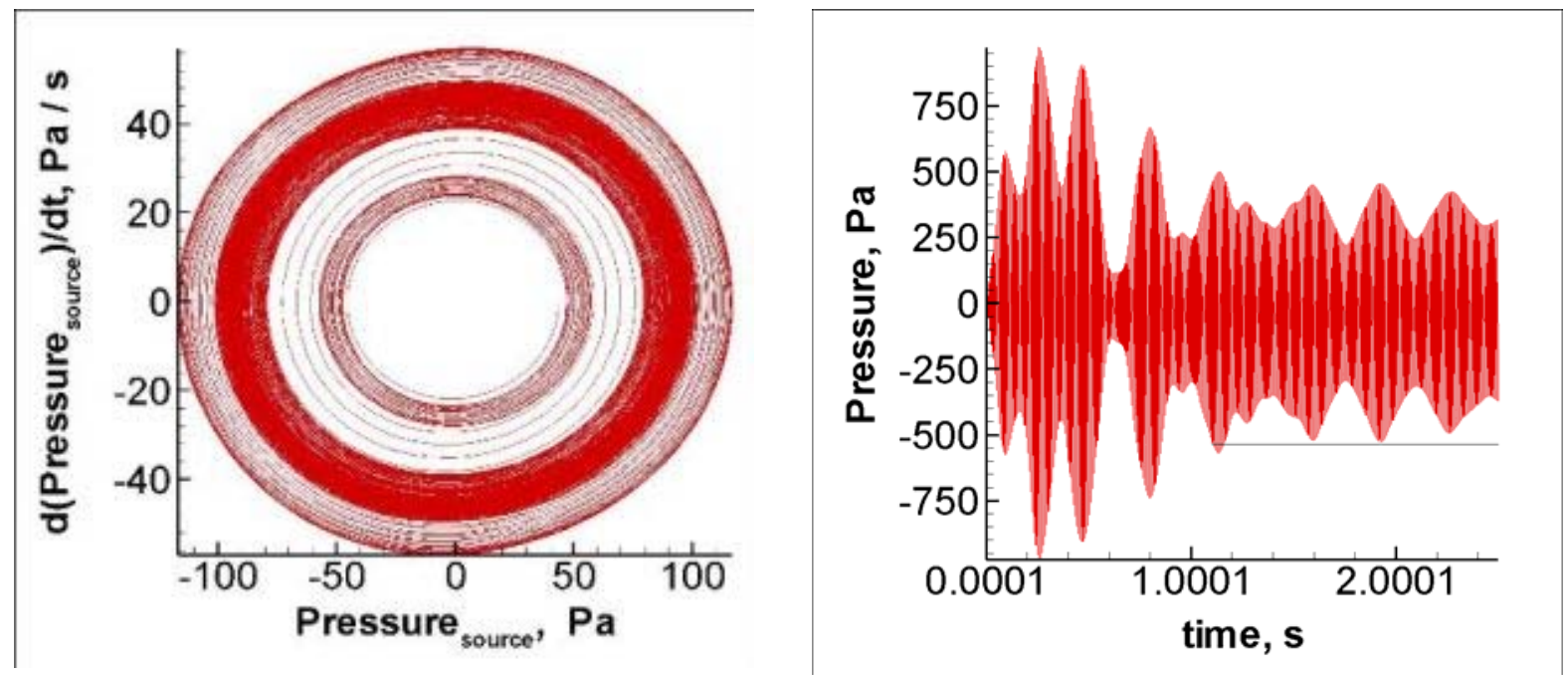

Figure 11. Numerical simulation of Case II, for $U=3 \mathrm{~m} / \mathrm{s}$ a) Phase plan of the source: limit circle; b) Pressure variation of the acoustic pipe
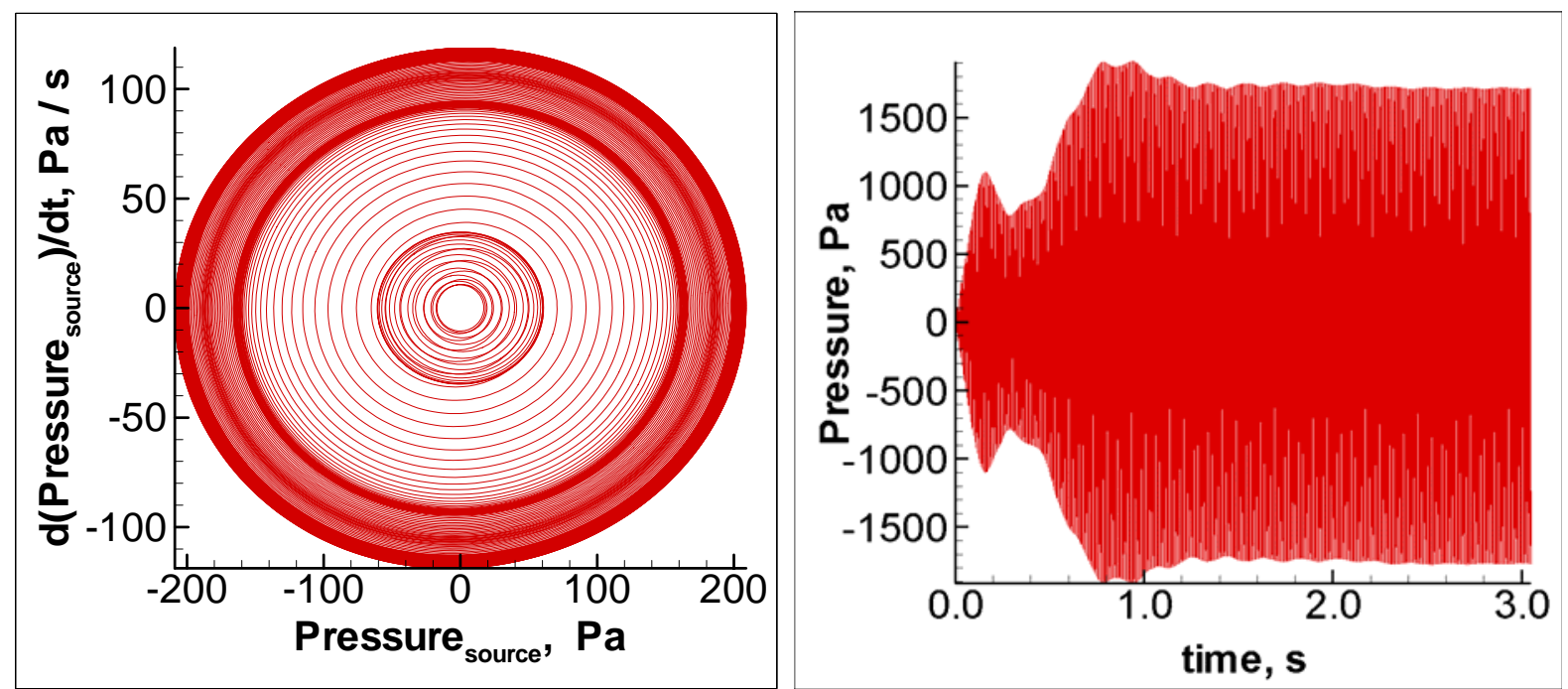

Figure 12. Numerical simulation of Case II, for $U=3.5 \mathrm{~m} / \mathrm{s}$ a) Phase plan of the source: limit circle; b) Pressure variation of the acoustic pipe. 


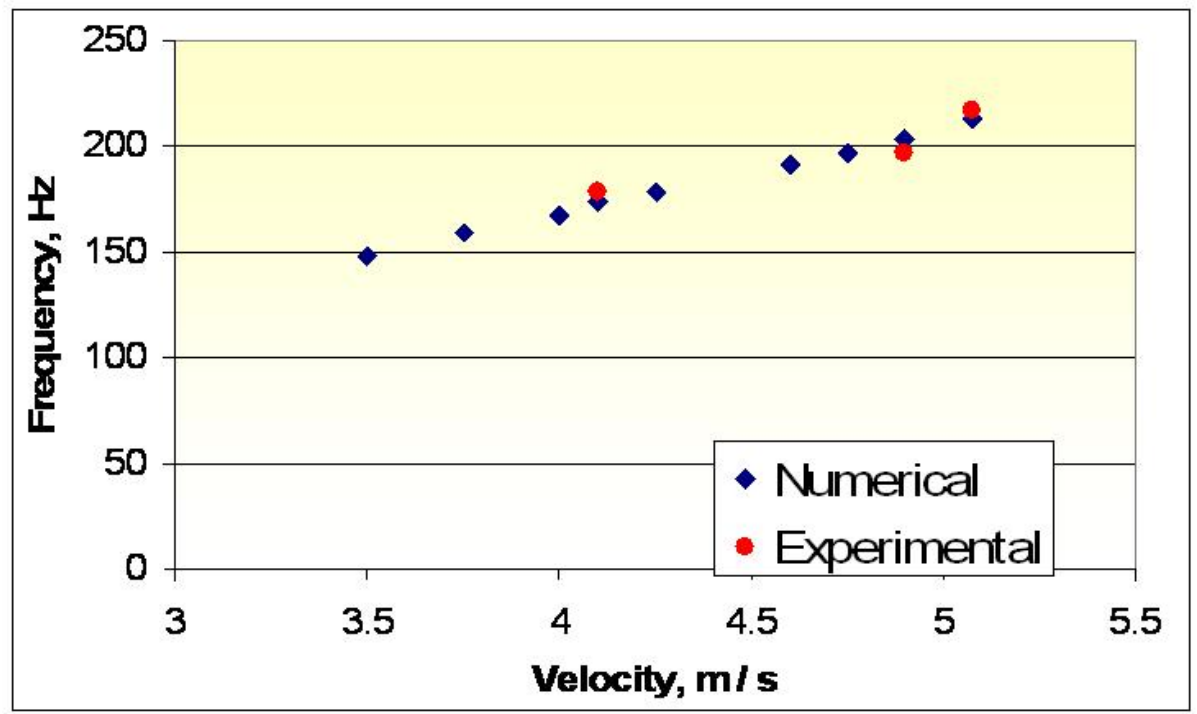

Figure 13. Evolution of frequency versus mean flow velocity in Case II 